\title{
Power Output and Efficiency of Beta-Emitting Microspheres
}

\author{
David Cheneler ${ }^{\mathrm{a}^{*}}$, Michael Ward ${ }^{\mathrm{b}}$ \\ a School of Engineering, Lancaster University, Lancaster, LA1 4YW, UK \\ b School of Engineering, Design and Manufacturing Systems, Birmingham City \\ University, Birmingham, B4 7XG, UK, E-Mail: michael.ward@bcu.ac.uk \\ * Corresponding author: E-Mail: d.cheneler@lancaster.ac.uk; Tel.: +44-1524-594- \\ 578; Fax: +44-1524-381-707.
}

\begin{abstract}
Current standard methods to calculate the dose of radiation emitted during medical applications by beta-minus emitting microspheres rely on an over-simplistic formalism. This formalism is a function of the average activity of the radioisotope used and the physiological dimensions of the patient only. It neglects the variation in energy of the emitted beta particle due to selfattenuation, or self-absorption, effects related to the finite size of the sphere. Here it is assumed the sphere is comprised of a pure radioisotope with beta particles being emitted isotropically throughout the material. The full initial possible kinetic energy distribution of a beta particle is taken into account as well as the energy losses due to scattering by other atoms in the microsphere and bremsstrahlung radiation. By combining Longmire's theory of the mean forward range of charged particles and the Rayleigh distribution to take into account the statistical nature of scattering and energy straggling, the linear attenuation, or self-absorption, coefficient for beta-emitting radioisotopes has been deduced. By analogy with gamma radiation transport in spheres, this result was used to calculate the rate of energy emitted by a betaemitting microsphere and its efficiency. Comparisons to standard point dose kernel formulations generated using Monte Carlo data show the efficacy of the proposed method. Yttrium-90 is used as a specific example throughout, as a medically significant radioisotope, frequently used in radiation therapy for treating cancer.
\end{abstract}

\section{Keywords}

Self-absorption; beta-decay; dosimetry; Y-90; P-33; Lu-177; microspheres; mass attenuation

\section{Introduction}

Beta-minus emitting radioactive microspheres are increasingly used in medical applications such as PET/SPECT imaging [1-4] and radiation therapy [5-12] as they often offer advantages over alternative methods such as chemoembolization and external beam radiation [13-14]. For instance, in radioembolization [6, 8, 15] or Selective Internal Radiation Therapy (SIRT) [16], 
radioactive microspheres are delivered to a tumour through the bloodstream via a catheter prior to an angiogram. The particles lodge in the tumour and emit radiation that kills the tumour. This method of cancer treatment is commonly used in conjunction with more well-established treatments such as chemotherapy. While this method offers advantages over external beam radiation, there is still a danger of complication due to extrahepatic deposition of the radioactive material [17-20]. To minimise the potential of further injury, the dose of radiation delivered to the patient needs to be well controlled [18, 21]. However, the absorbed dose, as described by a widely used formalism [7, 22, 23], is a function of the expected activity and pertinent physiological dimensions only, which does not include the variation in energy of the emitted electron due to self-attenuation effects related to the finite size of the particle.

There are many types of radionuclides used in medicine and generally are used as spheres of various sizes and compositions [24-29]. The energy of the electrons emitted by these radioactive microspheres can vary over several orders of magnitude and for lower energy electrons, energy loss within the microsphere can be significant. This effect can be compounded if the microspheres cluster during radioimmunotherapy [30-31].

The electrons lose energy as they travel through the microsphere due to interactions and collisions with atoms [32, 33]. During these interactions, atoms can absorb energy, slowing down the electron and deflecting it from its original trajectory. This is self-attenuation. The deflection of the electrons trajectory is mainly due to elastic scattering, inelastic scattering, ionization and Bremsstrahlung radiation. There are other scattering processes and loss mechanisms such as Moller scattering etc. but these are relatively unimportant [32]. Elastic scattering occurs due to Coulomb interactions between the electron and an atomic nucleus screened by the atomic electrons. Elastic scattering does not result in a loss of energy in the electron and leaves the atom undisturbed. During an inelastic interaction, or excitation, the incident electron interacts with atomic electrons. This can raise the atomic electron into an excited state while the original continues with reduced energy. During ionization, the incident electron knocks out one of the atomic electrons, thus ionising the atoms. Bremsstrahlung radiation can be emitted when the electron is briefly accelerated as it is being deflected. The accompanying energy loss in the form of photons is possibly significant only at relativistic energies, i.e. $>1 \mathrm{MeV}$ [33]. As the emitted electron passes through matter, it will interact with many atoms along its path. The electrons trajectory will therefore deviate from its original straight-line path, losing energy due to excitation and ionization and given enough material, eventually come to rest. Therefore, the true energy emitted by a radioactive particle is not just a function of its activity but of its size as well [34]. This is captured within a self-attenuation coefficient which is characteristic of the radioactive material. Measurement of this coefficient is non-trivial as many factors need to be taken into account. To this end, a number of researchers are still trying to generate accurate empirical relationships for the related mass attenuation coefficient albeit for non-radioactive absorbers [35, 36].

Calculations on the energy lost by beta particles have largely been focussed on aqueous media because of the important medical implications [37-39]. These models are generally formulated using either solutions of the transport equations [40, 41], Monte Carlo calculations [39, 42-43], or convolutions of tabulated data from experimental sources such as thin foil [44] or 
PET/SPECT measurements [45, 46]. Each of these methods have their advantages and limitations. Solving the transport equations, as is used here for instance, is a fast and robust method but without the previously mentioned mass attenuation coefficients and analytical solutions for the spherical geometry, requires significant numerical calculations [41]. Similarly, Monte Carlo methods have been very successfully used in dosimetry, but require substantial computational effort to generate sufficient data sets [42, 43]. Fortunately codes such as FLUKA [47] and Geant4 [48] are becoming increasingly robust and sophisticated and are being adapted for this purpose. Monte Carlo calculations are frequently used to generate 'point dose kernels' which are non-physical functions fitted to the numerical data to represent the energy absorbed by various, usually aqueous, media upon exposure to a point beta particle source. These kernels could then be used to numerically calculate the dose due to exposure to multiple or distributed sources. One of the most frequently cited references was by Berger [37] who used the ETRAN code to simulate monoenergetic electrons and produced dose point kernels that took into account multiple Coulomb scattering and energy transport by bremsstrahlung production. This work was extended by Prestwich et al. [41] who calculated the pose point kernels for six radionuclides of interest in nuclear medicine (Y-90, P-32, Cu-67, I-131, Re-186 and Re-188) considering only beta particle emission but took into account the full energy spectrum. More recently, Cross [49] proposed empirical calculations of doses delivered by point sources at a distance. Most of the codes used to generate the point source data are limited by the validity of the multiple diffusion theory at low energies and therefore are rarely applicable below 1-20 keV [50]. These point dose kernels are rarely amenable to analytical calculation unless they are in a convenient form [51, 52].

In this paper, the power emitted by a radioactive sphere, and hence its activity, is calculated. Expressions developed by Fermi [53, 54] for the energy distribution of the electron immediately after decay including correction factors that include the effect of Coulomb interactions are used to determine the initial activity. The deviation of the trajectory of the electrons caused by multiple scattering and the energy loss due to ionization and excitation are calculated assuming a continuum model whereby scattering processes are continuous and not discreet. This model is used to calculate the change in the energy distribution of the electrons as they travel through the material and by fitting an exponential model to this data, the selfabsorption coefficient is determined. By analogy with an analytical solution recently developed for a spherical gamma emitter by Atkinson and Brezovich [55], the total power output and efficiency of a beta-emitting microsphere is calculated. While the theory presented can be applied to any beta emitting sphere for any application, the specific case of a sphere composed of pure Yttrium-90, or Y-90, will be considered as an example. This is because Y-90 is a medically significant isotope of Yttrium frequently used in radiation therapy for treating cancer. Results are compared to point dose kernel formulations generated by fitting functional forms to tabulated Monte Carlo data which show the efficacy of the proposed method. 


\section{Theory}

During beta-minus decay, as considered here, a neutron converts to a proton causing two particles to be ejected from the nucleus: the electron and the antineutrino. In the case of Y-90, which has an atomic number, $Z$, of 39, the result is an isotope of Zirconium, Z-90 with an atomic number, $Z$ ', of 40 . The available kinetic energy released by the reaction is divided between the antineutrino and the electron. Therefore, the kinetic energy of the emitted electron, $T$, can take any value less than the maximum kinetic energy available, $Q$, given by [32]:

$$
Q=M_{1} c^{2}-M_{2} c^{2}-m_{e} c^{2}-m_{\bar{v}} c^{2}
$$

$M_{1}$ is the nuclear mass of the original parent nucleus, $M_{2}$ is the nuclear mass of the daughter nucleus after decay and $m_{e}$ is the mass of the electron. The mass of the antineutrino, $m_{\bar{v}}$, is vanishingly small. $c$ is the speed of light. For $\mathrm{Y}-90, Q=2.2798 \mathrm{MeV}$ [56]. To first order, the distribution of kinetic energies that emitted electrons that be expected to possess is given by Fermi's theory of beta decay as [32, 33]:

$$
N(T)=\frac{C}{c^{5}}\left(T^{2}+2 T m c^{2}\right)^{1 / 2}(Q-T)^{2}\left(T+m c^{2}\right)
$$

where $C=g^{2}\left|M_{f i}\right|^{2} /\left(2 \pi^{3} \hbar^{7} c\right), g$ is the weak interaction constant, $M_{f i}$ is the nuclear matrix element as defined in [32] and $\hbar$ is the reduced Planck's constant. $m$ is the mass of the electron (the subscript will be dropped hereafter). This expression neglects two important factors which causes it to deviate significantly from the experimentally observed distribution. The first factor arises from the Coulomb interaction between the electron and the daughter nucleus which was neglected in Eq. 2. The Coulomb interaction causes an attraction of the electron to the daughter nucleus resulting in more low-energy electrons. A correction for this can be added to Eq. 2 in the form of the Fermi function [53, 54]:

$$
F\left(Z^{\prime}, W\right)=\frac{2(1+S)}{((2 S) !)^{2}}(2 p \rho)^{2 S-2} e^{\pi \eta} \mid(S-1+i \eta) !^{2}
$$

where $S=\left(1-\alpha^{2} Z^{\prime 2}\right)^{1 / 2}, \rho=R /(\hbar / m c), R$ is the nuclear radius, $p$ is the electron momentum, $Z$ 'is the nuclear charge on the daughter nucleus, $\alpha$ is the fine structure constant and $\eta=Z^{\prime} e^{2} / \hbar v$ where $v$ is the speed of the electron far away from the nucleus. $W$ is the total energy (kinetic plus rest energy) corresponding to momentum $p$ in units of $m c^{2}$ and $m c$ respectively. In order to simplify calculations, Nilsson derived an expression with an error of one-half percent at most [57]:

$$
F\left(Z^{\prime}, W\right) \cong a \frac{W}{p}+\frac{C}{1+d / p^{2}}
$$


with $a=2 \pi \alpha Z^{\prime}, b=a /\left(1-e^{-a}\right), C=b-a$ and $d=\frac{1}{2}(b-1)$. The second factor arises from the 'forbiddenness' of the nuclear decay. A 'forbidden' decay does not mean the decay is impossible but rather is less probable than allowed decays and so may have a longer half-live. An allowed decay is described by eq. 2 which was derived by taking just the first term in the Taylor expansion of the wavefunctions describing the nucleus, antineutrino and electron during decay. When decays are forbidden, higher order terms need to be included as well, with the significance of each term being weighted by the proportion of experimentally observed decays with the pertinent half-life. For instance, $99.983 \%$ of Y-90 decays falls into the unique $1^{\text {st }}$ forbidden category [58]. Therefore, Eq. 2 needs to be further modified by a 'shape factor' which includes the higher order terms of the wavefunctions. In this case, the correction is [59]:

$$
S=\frac{1}{12}\left(p^{2}+q^{2}\right)\left[1+\left(\frac{b_{S}}{T}\right)\right]
$$

with $b_{S}=0.2 \sim 0.3$, taken in this case to be 0.25 . Note $S$ is in units of $\hbar=m=c=1 . q$ is the momentum of the antineutrino which can be given as:

$$
q=\frac{Q-T}{C}
$$

The energy distribution of Y-90 can therefore be given as:

$$
N_{C}(T)=N(T) F\left(Z^{\prime}, W\right) S
$$

This distribution is shown in Fig. 1:

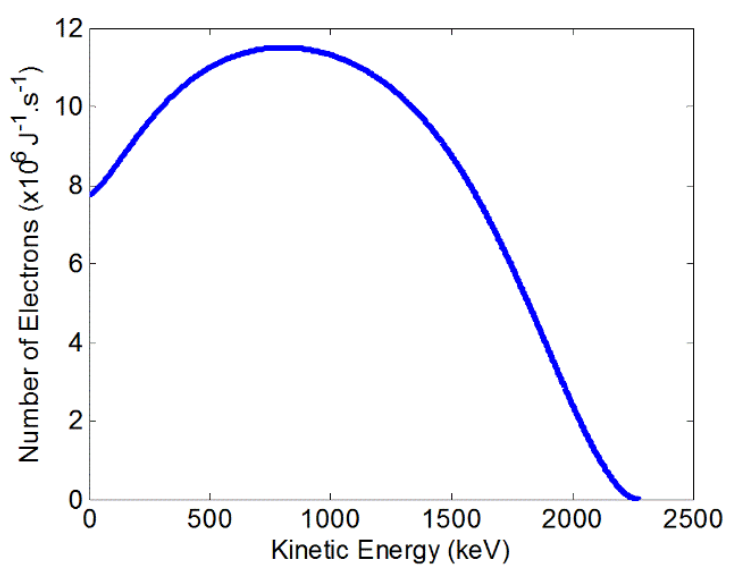

Fig. 1: Expected electron energy distribution for Y-90.

Note the average kinetic energy calculated from this spectrum is $927.4 \mathrm{keV}$, which is very close to $926.7 \mathrm{keV}$ as given in [58], and with $\left|M_{f i}\right|^{2}$ set at $5.56 \times 10^{-5}$ in eq. 2, the half-life can be calculated to be 2.6684 days as expected [56]. As the emitted electrons travel through the material, they will interact with atomic electrons through Coulomb scattering. This scattering causes the emitted electron to follow erratic paths so that the range (defined as the linear 
distance of penetration into the material [32]) is very different to the actual length of the path taken. The electron, which initially may be travelling at relativistic speeds, may, as a result of collisions with atomic electrons, experience rapid changes of direction and speed. This results in high accelerations, and being charged, the electrons emit electromagnetic energy or bremsstrahlung radiation. Therefore two sources of energy dissipation must be considered, that due to momentum exchange during collisions and that due to radiation. Bethe derived expressions for both cases in the form of energy loss per unit length [32]:

$$
\begin{gathered}
\left(\frac{d T}{d x}\right)_{c}=\left(\frac{e^{2}}{4 \pi \varepsilon_{0}}\right) \frac{2 \pi N_{A} Z \rho}{m c^{2} \beta^{2} A}\left[\ln \left(\frac{T\left(T+m c^{2}\right)^{2} \beta^{2}}{2 I^{2} m c^{2}}\right)+\left(1-\beta^{2}\right)\right. \\
\left.-\left(2 \sqrt{1-\beta^{2}}-1+\beta^{2}\right) \ln 2+\frac{1}{8}\left(1-\sqrt{1-\beta^{2}}\right)^{2}\right] \\
\left(\frac{d T}{d x}\right)_{r}=\left(\frac{e^{2}}{4 \pi \varepsilon_{0}}\right) \frac{Z^{2} N_{A}\left(T+m c^{2}\right) \rho}{137 m^{2} c^{4} A}\left[4 \ln \left(\frac{2\left(T+m c^{2}\right)}{m c^{2}}\right)-\frac{4}{3}\right]
\end{gathered}
$$

where $\mathrm{x}$ is in the direction of the electrons path. $Z$ and $A$ are the atomic number and atomic mass of the material the electron is travelling through respectively, which is taken to be pure Y-90 in this case. $e$ is the charge of an electron, $N_{A}$ is Avogadro's number, $\rho$ is the density of Y-90, $\beta=v / c$ and $I$ is the ionization energy of Y-90 taken to be $379 \mathrm{eV}$ [60]. The subscripts $c$ and $r$ stand for the energy losses due to collisions and bremsstrahlung radiation respectively. The total energy loss is the sum of these two contributions:

$$
\frac{d T}{d x}=\left(\frac{d T}{d x}\right)_{c}+\left(\frac{d T}{d x}\right)_{r}
$$

The relative contributions of these energy losses is shown in Fig. 2.

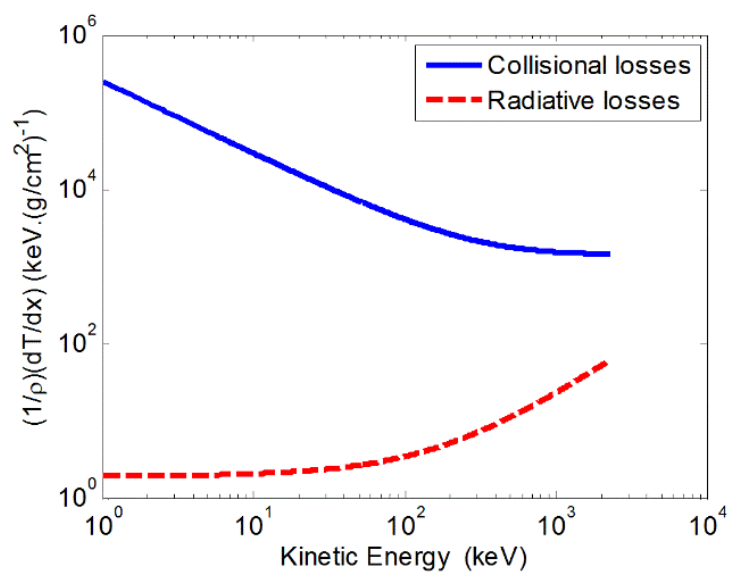

Fig. 2: Expected energy loss by electrons in Y-90. 
In reality, electrons lose energy at more or less than the average rate given by Eq. 10. Assuming that electrons lose energy at this average rate is known as the continuous slowing down approximation and permits investigation without resorting to statistical analysis. As well as losing energy, this scattering naturally leads to a change in direction, an effect which increases in importance for lower energy electrons. The path taken by the electron is akin to a directed random walk with multiple scattering often dominating the electrons motion. Initially, while still at high energies, the scattering causes a slow divergence in angle from the initial path. At lower energies the angle of scattering is larger and the deviation from the original path can be significant. Using the Williams theory of scattering which is based on Rutherford's theory for individual electron-atom scattering, the rate of change of the mean square scattering angle, $\left\langle\theta^{2}\right\rangle$, can be shown to be [33]:

$$
\frac{d\left\langle\theta^{2}\right\rangle}{d x}=\frac{8 \pi N_{A} Z(Z+1) r_{o}^{2}}{A \gamma^{2} \beta^{4}} \ln \left(\frac{\theta_{\max }}{\theta_{s}}\right)
$$

where $\gamma=1+T / m c^{2}$ and $\theta_{\max }$ is the maximum angle that is considered 'small' during a single scattering. $\theta_{s}$ can be considered the smallest angle that a neutral atom can scatter an electron due to the shielding effects of the atomic electrons and the finite size of the atom which negates the singularities in Rutherford's theory. There are a number of ways to choose $\theta_{s}$ and $\theta_{\max }$. Here the same approach as taken in $[33,61]$ is used, i.e.:

$$
\theta_{\max }=\min \left[1, \frac{280}{\gamma \beta A^{1 / 3}}\right]
$$

and

$$
\theta_{s}^{2}=\left(\frac{\alpha}{\beta \gamma}\right)^{2} \frac{Z^{2 / 3}}{(0.885)^{2}}\left[1.13+3.76\left(\frac{\alpha Z}{\beta}\right)^{2}\left(\frac{T}{T+m c^{2}}\right)^{1 / 2}\right]
$$

The rate of increase of the mean square scattering angle with respect to the decrease in kinetic energy of the electron can be defined as:

$$
\frac{d\left\langle\theta^{2}\right\rangle}{d T}=-\frac{d\left\langle\theta^{2}\right\rangle / d x}{|d T / d x|}
$$

Longmire [62] was able to gain an analytical expression for Eq. 14, but neglected the radiative losses. Here Eq. 14 is solved numerically to find the mean square scattering angle. In the same publication, Longmire also extended Williams theory in order to calculate the mean forward range which is the mean distance travelled in the direction of an initially collimated beam by a population of electrons with the same initial energy. This theory gives the range of an electron with initial energy $T_{0}$ undergoing multiple scattering and experiencing collisional and radiative energy loss as [33, 62]: 


$$
R=\int_{0}^{T_{0}} \frac{1}{\eta}\left|\frac{d x}{d T}\right| d T
$$

where $\eta=1+0.5\left\langle\theta^{2}\right\rangle$. In Fig. 3, the effect of scattering is clear. An electron emitted with the maximum allowed initial kinetic energy from within a large body of Y-90 will follow a path with an average length of $2.9 \mathrm{~mm}$. However, as this path will been deflected many times due to scattering, the mean distance the electron will have travelled in the direction of its initial trajectory is just c.a. $700 \mu \mathrm{m}$.
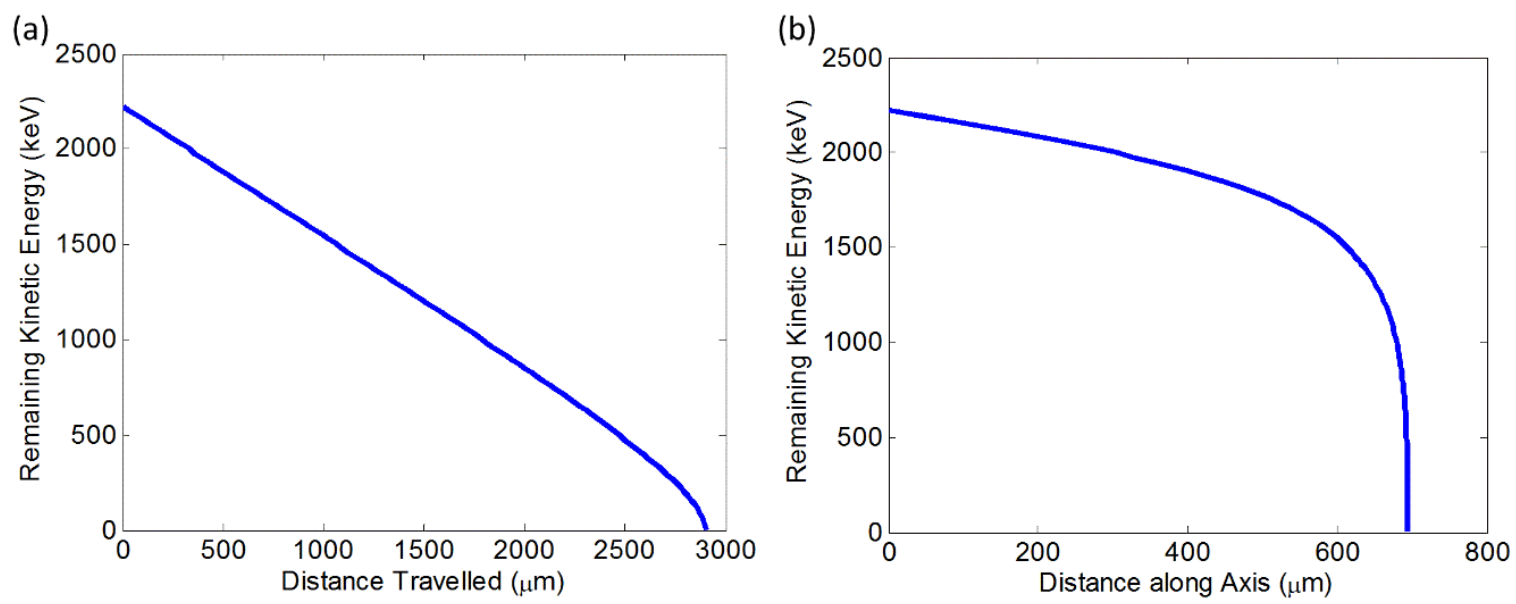

Fig. 3: (left) the loss of kinetic energy of a beta particle with initial kinetic energy $Q$ as a function of the true expected distance travelled and (right) the loss of kinetic energy of a beta particle with initial kinetic energy $Q$ as a function of the expected projected distance travelled in the direction of the initial trajectory.

From Eq. 15, the following range-energy relationship for the beta particles emitted in Y-90 can be calculated:

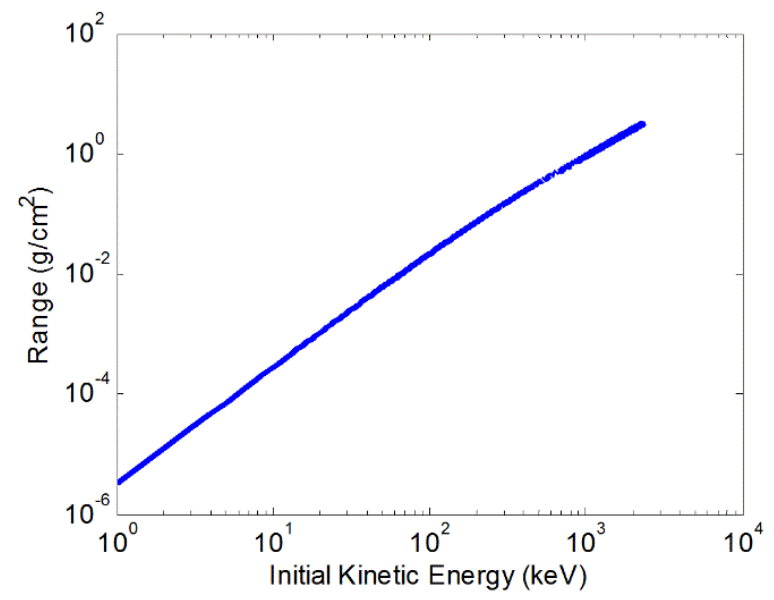

Fig. 4: Range-energy relationship for the beta particles in Y-90.

As mentioned above, the energy loss of a beta particle is a probabilistic process and so an actual beta particle may travel somewhat more or less than given by Eq. 15. To account for this, it is 
assumed that the differential range distribution of the beta particles obeys the Rayleigh distribution, which can be given as [63]:

$$
p(x) d x=\left(\frac{x}{R^{2}}\right) \exp \left(-\frac{x^{2}}{2 R^{2}}\right) d x
$$

where $R$ is the mean range as given by Eq. 15 and $x$ is the thickness of the material. The probability the beta particle will travel at least a distance $x$ is therefore:

$$
P(x)=\int_{x}^{\infty} p(x) d x=\exp \left(-\frac{x^{2}}{2 R^{2}}\right)
$$

As the beta particle are emitted with a distribution of possible energies, as given by Eq. 7, they will have a distribution of possible mean ranges as shown in Fig. 4. Therefore, the energy distribution is divided into groups described by a probability mass function so that the $\mathrm{i}^{\text {th }}$ group of beta particles can be treated as a monoenergetic beam with kinetic energy:

$$
T_{i}=\frac{Q}{k}\left(i-\frac{1}{2}\right)
$$

where $k$ is the total number of groups. The relative emitted intensity, i.e. the proportion of beta particles that are emitted with kinetic energy $T_{i}$ can be shown to be:

$$
I_{i}=\frac{\int_{T_{i-1 / 2}}^{T_{i+1 / 2}} N_{C}(T) d T}{\int_{0}^{Q} N_{C}(T) d T}
$$

In this way, the energy spectrum can be represented thus:

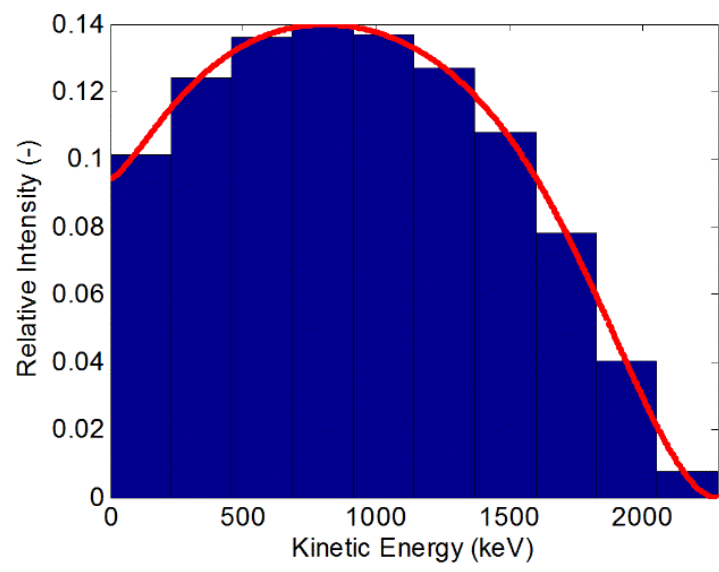

Fig. 5: Probability mass function (PMF) of the relative intensity of beta particles for a given kinetic energy $T_{i}$. The red line denotes the continuous relative energy distribution 


\section{given by Eq. 7. Note $k=10$ for this figure, however it was 1000 during subsequent calculations.}

The relative intensity $n_{i}(x)$ of electrons from the $\mathrm{i}^{\text {th }}$ group passing through the material with thickness $x$ is:

$$
n_{i}(x)=I_{i} \exp \left(-\frac{x^{2}}{2 R_{i}^{2}}\right)
$$

Fitting an exponential curve of the form:

$$
F i t=C \exp (-\mu x)
$$

where $C$ is an arbitrary constant and $\mu$ is the linear attenuation coefficient (in $\mathrm{cm}^{-1}$ ), to the lower portion of Eq. 20 (it was found that fitting to $n_{i}(x)<0.8 I_{i}$ gave the best fit) permits the calculation of $\mu$. Given the linear attenuation coefficient was calculated with Y-90 as the absorber, this is by definition also the self-attenuation coefficient for Y-90. As is common practice, dividing $\mu$ by the density of yttrium gives the mass attenuation coefficient in units of $\mathrm{cm}^{2} \mathrm{~g}^{-1}$. The result is shown in Fig. 6. The calculated mass attenuation coefficient of Y-90 can be compared to a power-law approximation of the same form used by Thontadarya for an aluminium absorber [64]:

$$
\left(\frac{\mu}{\rho}\right)=\frac{14.5}{T^{1.58}}
$$

where $T$ is in $\mathrm{MeV}$. The relative error between that calculated using Eq. 21 and the approximate expression given by Eq. 22 is less than 5\% for energies above $10 \mathrm{keV}$. Below 10 $\mathrm{keV}$, the relative error increases to $15 \%$

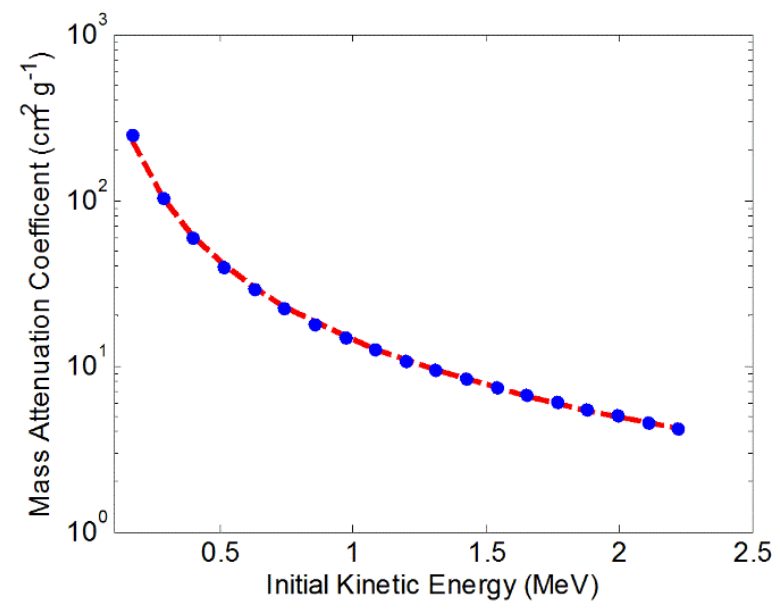

Fig. 6: The mass attenuation coefficient of electrons in Yttrium.

Y-90 has a theoretical activity density, or number of decay events per unit volume per second, of $9 \times 10^{5} \mathrm{GBq} / \mathrm{cc}$ and a theoretical power density, or energy produced per unit volume per 
second, of $13.4 \mathrm{~kW} / \mathrm{cc}$. These densities are very high for beta sources and are due to the materials high energy decay and relatively short half-life. Denoting the activity density as $A_{M}$, it can be shown that the total flux rate of beta particles at the surface of a self-attenuating sphere is [55]:

$$
\Phi=A_{M} \sum_{i=1}^{k} \frac{\pi R^{2} I_{i}}{\mu_{i}}\left\{1+\frac{1}{\mu_{i} R} e^{-2 \mu_{i} R}-\frac{1}{2 \mu_{i}^{2} R^{2}}\left[1-e^{-2 \mu_{i} R}\right]\right\}
$$

where $R$ is the radius of the sphere. This result gives the number of electrons that will enter a tissue should a sphere of radius $R$ comprised of pure Y-90 be encapsulated in it. As these electrons will have been emitted with a distribution of energies, it is necessary to also know the rate of energy entering the tissue. This is given in Eq. 24:

$$
P=A_{M} \sum_{i=1}^{k} \frac{\pi R^{2} T_{i} I_{i}}{\mu_{i}}\left\{1+\frac{1}{\mu_{i} R} e^{-2 \mu_{i} R}-\frac{1}{2 \mu_{i}^{2} R^{2}}\left[1-e^{-2 \mu_{i} R}\right]\right\}
$$

In Fig. 7, the results of Eqtns. 23 and 24 in terms of the number of electrons per second emitted from spheres of increasing radii and the associated power emitted are shown. Also shown are the number of electrons and power initially generated through radioactive decay in these spheres. As can be seen the number of electrons and power initially generated increase with the cube of the radius or volume of the sphere as expected. However, the number of electrons and the associated power that actually reach the spheres surface and is emitted increases at a slower rate. This is due to the attenuation and its effects are shown more clearly in Fig. 8 . The efficiency of the radioactive microspheres is defined in this instance as the power emitted divided by the power initially generated. As can be seen, when the spheres reach c.a. $940 \mu \mathrm{m}$, the efficiency has dropped to $50 \%$ meaning that half the energy available has been absorbed by the sphere itself. Indeed at sphere radii comparable to the mean range of the electrons (c.a. $185 \mu \mathrm{m})$, the losses are significant.

(a)

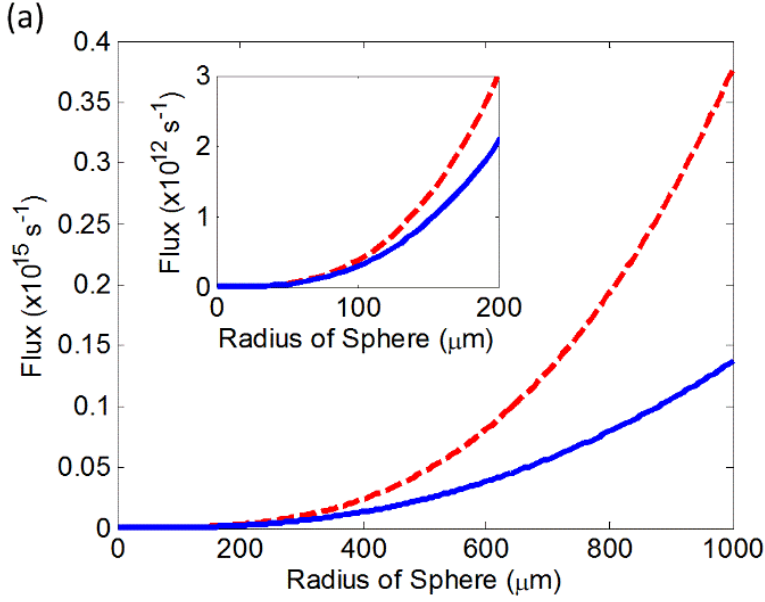

(b)

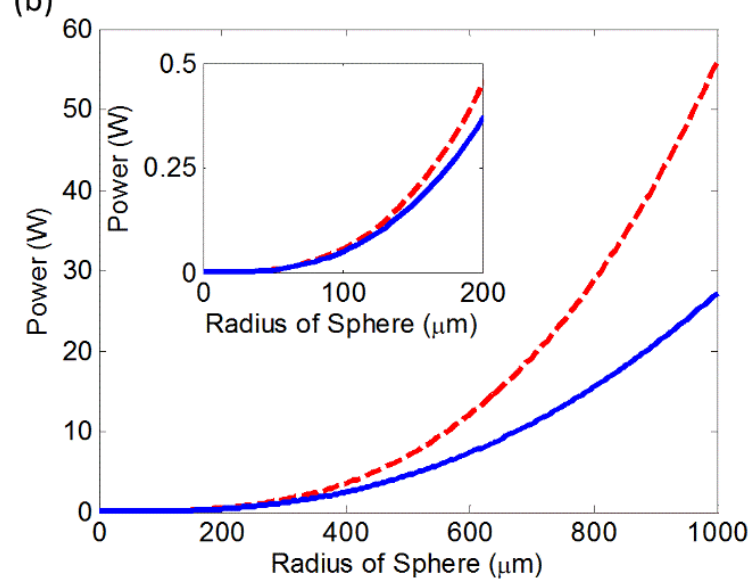

Fig. 7: (a) The number of electrons initially generated and consequently emitted from the surface of spheres composed of Y-90 of increasing radii and (b) the associated power generated and emitted from the sphere. Insets: close up of response of smaller diameter 
spheres. In all cases the dashed red line represents beta particles generated within the sphere and the solid blue line represents beta particles emitted from surface of sphere.

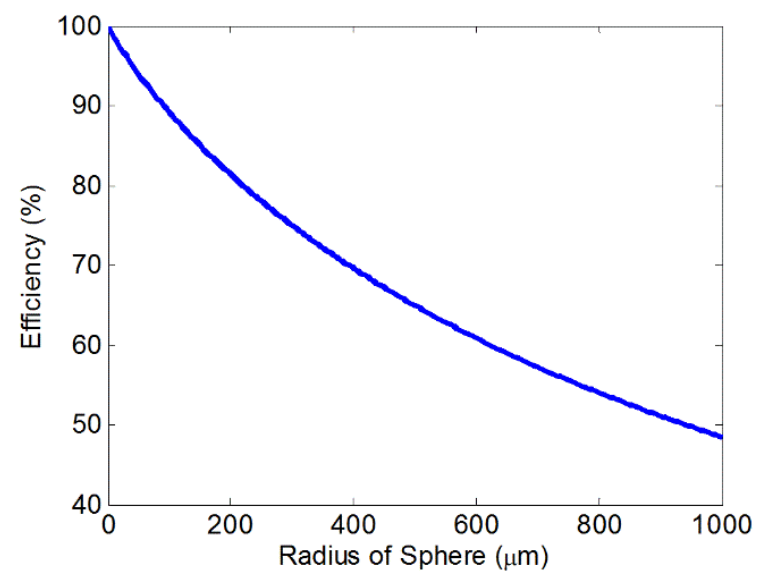

Fig. 8: The efficiency of microspheres composed of Y-90. Note the efficiency decreases with increasing sphere radii due to self-attenuation.

Therefore, for the case of a microsphere comprised entirely of Y-90 with beta particles being randomly produced homogeneously and isotropically throughout the whole sphere, there is less than a $1 \%$ error in the expected power output if self-absorption is neglected if the sphere radius is less than $6.5 \mu \mathrm{m}$. This rises to a $5 \%$ error if the sphere radius is $40 \mu \mathrm{m}$ and $10 \%$ for a 91.5 $\mu \mathrm{m}$ sphere radius. It is unlikely that spheres larger than this will be used in medical applications. Also in radioembolization therapies [1,8], it is currently far more common for the microsphere to be comprised of Y-90 nanoparticles homogeneously dispersed in a glass or resin matrix. The above model can account for this, if it is assumed that all losses considered in eq. 10 are due to interactions with a single type of atom. Otherwise, the theory would have to be generalised to take into account the exact composition of the material. If it is assumed that the Y-90 is distributed at low density in glass, $\mathrm{SiO}_{2}$, in such a manner that any beta particle produced will only interact with the glass, which is assumed to have density $2650 \mathrm{~kg} / \mathrm{m}^{3}$ and average atomic number 10 and mass 20u, then the effects of self-absorption can be modelled as above. The results are shown below in Fig. 9(a). As expected, beta particles lose less energy as they travel a given distance in glass as they would in Y-90. In this case, the effect of neglecting selfabsorption within the microsphere is less than $1 \%$ for spheres with a radius less than $25 \mu \mathrm{m}$, which is true for most commonly used glass spheres [8]. It is expected that resin spheres will have a comparable response. 
(a)

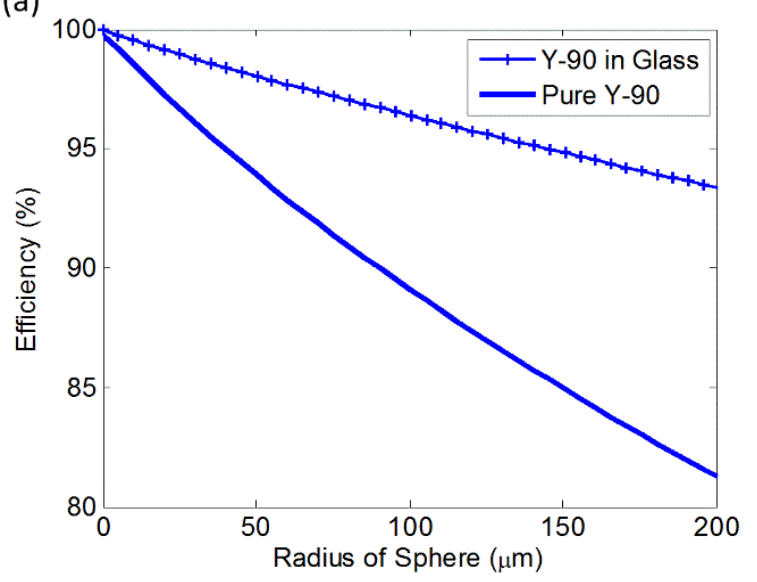

(b)

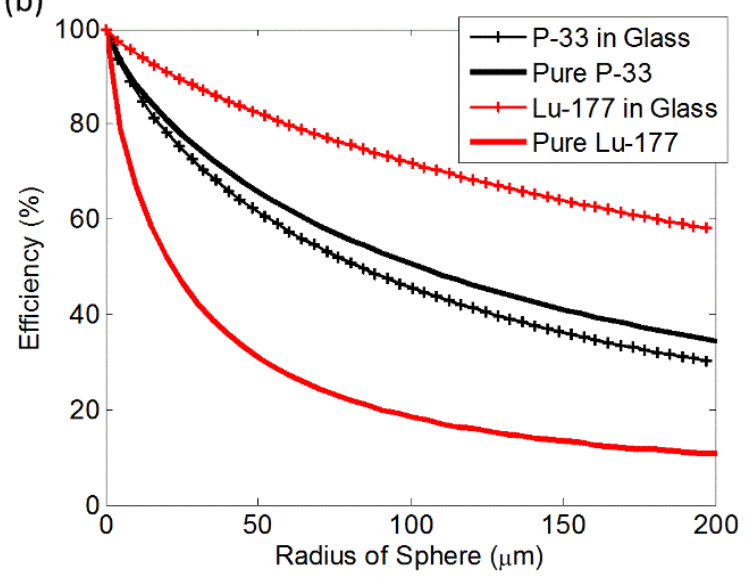

Fig. 9: (a) The efficiency of a microsphere comprised of pure Y-90 (blue solid line) compared to microspheres comprised of mostly glass with Y-90 as a beta source (blue crossed line). (b) The efficiencies of microspheres comprised of pure P-33 (black solid line) and of pure Lu-177 (red solid line) compared to microspheres comprised of mostly glass with P-33 (black crossed line) and Lu-177 (red crossed line) as a beta source.

However, as also seen in Fig. 9(b), while self-absorption can be neglected for Y-90 microspheres of a size used in most medical applications, this is not necessarily true for other medically relevant radioisotopes. This is because Y-90 emits high-energy beta particles. For comparison, as shown in Fig. 9, lutetium-177 (Lu-177) emits beta-minus particles of maximum energy $498 \mathrm{keV}$ (average energy $0.149 \mathrm{keV}$ ) has a half-life of 6.73 days and is used for treating micro-metastatic disease and bone pain palliation [65]. For a microsphere comprised of pure Lu-177, the self-absorption effects are much more pronounced with $10 \%$ losses expected for spheres with a $2 \mu \mathrm{m}$ radius and $50 \%$ losses for spheres with a $22 \mu \mathrm{m}$ radius. Similarly, phosphorus-33 (P-33) emits beta-minus particles of maximum energy $249 \mathrm{keV}$ (average energy $0.085 \mathrm{keV}$ ) has a half-life of 25.4 days and is used for nucleotide labelling [66]. P-33 will deposit $10 \%$ of its energy within a sphere of radius $8.5 \mu \mathrm{m}$ and $50 \%$ of its energy within a sphere of radius $100 \mu \mathrm{m}$. Therefore, the size of the microsphere can have an important effect on the actual energy emitted by a radionuclide. As mentioned above, it is common for radioisotopes to be encapsulated in glass or resin when used in radioembolization therapies. As for Y-90, the low density and atomic mass of the glass means that in the case of Lu-177, the effects of self-absorption are less pronounced in an encapsulated distributed source than for a single material source, with a sphere radius of $23 \mu \mathrm{m}$ needed before a loss of $10 \%$. However, in the case of P-33, the situation is different. As the density of P-33, taken to be $1820 \mathrm{~kg} / \mathrm{m}^{3}$, is less than glass, the self-absorption is actually higher when the microsphere is comprised of P33 distributed in a glass matrix with $10 \%$ energy loss within a radius of $7 \mu \mathrm{m}$.

As discussed in the introduction, there have been several models formulated to calculate dose absorbed by various media in different geometries. The most frequently used is arguably that calculated by Berger [37, 50, 67] for a uniform volume distribution of sources emitting monoenergetic electrons with initial energy $E_{0}$. In this formulation, the energy absorbed by a sphere of radius $R$ due to a distributed source is given by: 


$$
\phi=\frac{1}{r_{0}} \int_{0}^{2 R}\left[1-1.5\left(\frac{x}{2 R}\right)+0.5\left(\frac{x}{2 R}\right)^{3}\right] F\left(\frac{x}{r_{0}}, E_{0}\right) d x
$$

where $F\left(x / r_{0}, E_{0}\right)$ is the normalized 'point dose kernel' which represents the fraction of energy emitted by a monoenergetic point source absorbed within a spherical layer with a thickness $d x$ at a distance $x$ from the source. $r_{0}$ is the maximum range of the electron with initial energy $E_{0}$ and is used a normalisation constant. The normalised point kernel was taken from the work of Prestwich et al. [41] who fitted a non-physical function to data generated using Monte Carlo methods. Fitting data for Y-90 was supplied in [41] and used directly in Eq. 25. In [41] it was shown that the average deviation between the numerical data and the fitted function was within $2 \%$. Interestingly, Prestwich et al. compared their data against to that calculated by Spencer [40] who solved transport equation in a manner comparable to the method proposed here. Prestwich et al. showed that for electrons with energy below $50 \mathrm{keV}$, the dose predicted was overestimated by $6 \%$ by the transport equation and underestimated by 6 to $12 \%$ for energies above $0.5 \mathrm{MeV}$. It was noted that discrepancies were mainly due to Spencer neglecting bremsstrahlung and energy straggling effects, which are both included here. Neither extended the theory for spherical sources. While Prestwich et al. data used Y-90 as an electron source, it assumes, as is common practice, that the energy dissipation is through interaction with water. Scaling Eq. 25 in a manner suggested by Cross [68] to take into account the larger atomic number of Y-90, the power emitted by a Y-90 sphere can be calculated. The comparison between the point-kernel formulation and the model proposed here is shown in Fig. 10. The maximum deviation between the two models over the $0-1 \mathrm{~mm}$ sphere radius range is $7.5 \%$ with an average error of $4.4 \%$. This demonstrates that the proposed model can give accurate results for the power generated by nuclear microspheres without resorting to Monte Carlo calculations or curve-fitting/interpolation of tabulated data.

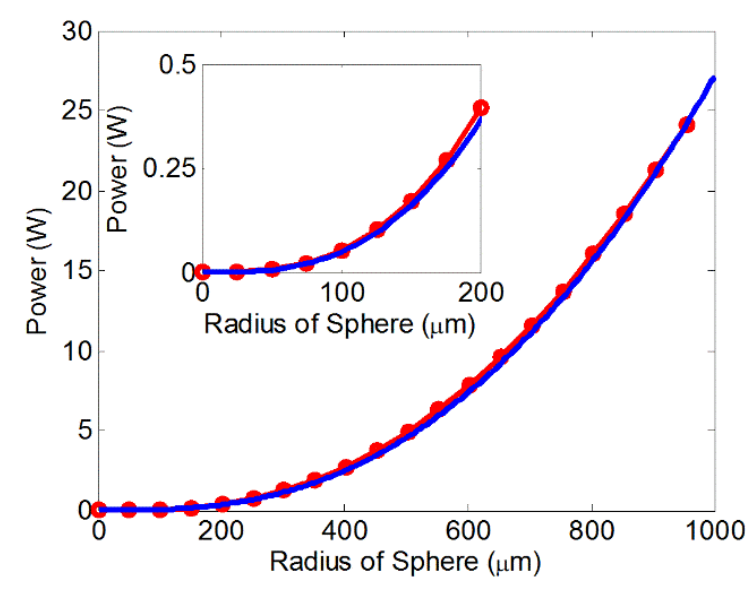

Fig. 10: Comparison between power generated by Y-90 microsphere of various radii calculated using the proposed model (blue solid line) as shown in Fig. 7(b) and that calculated using the point dose kernel approach given by Eq. 25 (circled red line). Inset: close up of response of smaller diameter spheres. 


\section{Conclusion}

In this paper, the effect of the finite size of beta producing radioactive microspheres on the rate of energy emitted and their efficiency has been shown. By utilising a continuous approximation approach, the energy losses that a beta particle experiences as it traverses the radioactive microsphere has been calculated. This result was combined with energy straggling effects by assuming a Rayleigh distribution which took into account the statistical nature of the actual range of the electron to calculate the mass attenuation coefficient, which in this case is the selfattenuation coefficient. By using an analogy with spherical gamma emitters, the flux and power output at the surface of beta emitting microspheres was given. In order to give a numerical example, the specific case of yttrium-90, a medically important beta source, was considered. Thus the self-attenuation coefficient for Y-90 was calculated and it was shown that the energy absorbed by the sphere itself becomes significant at sphere radii comparable to the mean range of the beta particles. Results shown for Lu-177 and P-33 show that the self-attenuation effect is important for some radionuclides and medically applicable microsphere radii. It is shown that the standard practice of encapsulating beta sources in a glass matrix has a profound effect on the self-attenuation coefficient for the microsphere, with the energy loss actually being increased if the density of the matrix is higher than that of the pure beta source. Using the method suggested by this paper, the dose supplied by beta-emitting microspheres can now be calculated far more accurately, reducing the risk of further health issues caused by over/under exposure.

\section{Acknowledgements}

This work was funded by the Engineering and Physical Sciences Research Council (EPSRC) (Grant No. EP/J01446X/1).

\section{References}

[1] Pasciak, A. S., Bourgeois, A. C., and Bradley, Y. C. (2014), A Comparison of Techniques for 90Y PET/CT Image-Based Dosimetry Following Radioembolization with Resin Microspheres. Frontiers in Oncology, Vol. 4, 121

[2] Elschot, M., et al. (2013), Quantitative comparison of PET and Bremsstrahlung SPECT for imaging the in vivo yttrium-90 microsphere distribution after liver radioembolization. PLoS One, Vol. 8, No. 2, e55742

[3] D’Arienzo, M., et al. (2012), 90Y PET-based dosimetry after selective internal radiotherapy treatments. Nuclear medicine communications, Vol. 33, No. 6, pp. 633-640

[4] Kao, Y. H., et al. (2012), Image-guided personalized predictive dosimetry by arteryspecific SPECT/CT partition modeling for safe and effective 90Y radioembolization. Journal of Nuclear Medicine, Vol. 53, No. 4, pp. 559-566

[5] Houle, S., et al. (1989), Hepatocellular carcinoma: pilot trial of treatment with ${ }^{90} \mathrm{Y}$ microspheres, Radiology, Vol. 172, pp. 857-860 
[6] Martin, L. K., et al., (2012), Yttrium-90 Radioembolization as salvage therapy for colorectal cancer with liver metastases. Clinical colorectal cancer, Vol. 11, No. 3, pp. 195-199

[7] Salem R. and Thurston, K. G., (2006), Radioembolization with ${ }^{90}$ yttrium microspheres: a state-of-the-art brachytherapy treatment for primary and secondary liver malignancies_-part 1: technical and methodologic considerations,” Journal of Vascular and Interventional Radiology, Vol. 17, No. 8, pp. 1251-1278

[8] Kennedy, A., et al., (2007), Recommendations for radioembolization of hepatic malignancies using yttrium-90 microsphere brachytherapy: a consensus panel report from the radioembolization brachytherapy oncology consortium. International Journal of Radiation Oncology, Biology, Physics, Vol. 68, No. 1, pp. 13-23

[9] Dadachova, E., et al., (2002), Rhenium-188 as an alternative to Iodine-131 for treatment of breast tumors expressing the sodium/iodide symporter (NIS). Nuclear medicine and biology, Vol. 29, No. 1, pp. 13-18

[10] Shukla, R., et al., (2012), Laminin receptor specific therapeutic gold nanoparticles (198AuNP-EGCg) show efficacy in treating prostate cancer. Proceedings of the National Academy of Sciences, Vol. 109, No. 31, pp. 12426-12431

[11] Kim, T. K., and Burgess, D. J., (2002), Pharmacokinetic characterization of 14C-vascular endothelial growth factor controlled release microspheres using a rat model. Journal of pharmacy and pharmacology, Vol. 54, No. 7, pp. 897-905

[12] Chanda, N., et al., (2010), Radioactive gold nanoparticles in cancer therapy: therapeutic efficacy studies of $\mathrm{GA}^{198}$ AuNP nanoconstruct in prostate tumor-bearing mice. Nanomedicine: Nanotechnology, Biology and Medicine, Vol. 6, No. 2, pp. 201-209

[13] Hoffmann, R. T., et al., (2011), Arterial therapies of non-colorectal cancer metastases to the liver (from chemoembolization to radioembolization). Abdominal imaging, Vol. 36, No. 6, pp. 671-676

[14] Gates, V. L., (2007), Radioembolization with Yttrium-90 microspheres: review of an emerging treatment for liver tumors, Vol. 3, No. 1, pp.73-81

[15] Vente, M. A., et al., (2009), Yttrium-90 microsphere radioembolization for the treatment of liver malignancies: a structured meta-analysis. European radiology, Vol. 19, No. 4, pp. 951-959

[16] Stubbs, R.S. and Cannan, R.J., (2002), Selective internal radiation therapy with yttrium90 microspheres for primary and metastatic cancer confined to the liver, N. Habib (Ed.), Multi-Treatment Modalities of Liver Tumours, Kluwer Academic/Plenum Publishers, London pp. 305-321

[17] Crowder, C. D., et al., (2009), Selective internal radiation therapy-induced extrahepatic injury: an emerging cause of iatrogenic organ damage. The American journal of surgical pathology, Vol. 33, No. 7, pp. 963-975

[18] Domínguez-Gadea, L., and Cerezo, L. (2011). Decontamination of radioisotopes. Reports of Practical Oncology \& Radiotherapy, Vol. 16, No. 4, pp. 147-152

[19] Riaz, A., et al., (2009), Complications following radioembolization with yttrium-90 microspheres: a comprehensive literature review. Journal of Vascular and Interventional Radiology, Vol. 20, No. 9, pp. 1121-1130 
[20] Aydarous, A. S., (2008), Calculated effects of backscattering on skin dosimetry for nuclear fuel fragments. Radiation protection dosimetry, Vol. 130, No. 2, pp. 141-148

[21] Garin, E., et al., (2011). Utility of quantitative ${ }^{99 m}$ Tc-MAA SPECT/CT for ${ }^{90}$ yttriumlabelled microsphere treatment planning: calculating vascularized hepatic volume and dosimetric approach. International journal of molecular imaging, 398051

[22] Jones, E. and Mallard, J. R., (1963). The Dosimetry of Yttrium-90 Sources Suitable for Pituitary Implantation: Comparison of Theoretical and Experimental Results. Physics in Medicine and Biology, Vol. 8, No. 1, pp. 75-83

[23] Mo, L., et al. (2005). Development of activity standard for ${ }^{90} \mathrm{Y}$ microspheres. Applied radiation and isotopes, Vol. 63, No. 2, pp. 193-199.

[24] Dezarn, W. A., (2008), Quality assurance issues for therapeutic application of radioactive microspheres. International Journal of Radiation, Oncology, Biology, Physics, Vol. 71, No. 1, pp. S147-S151

[25] Brouwers, A. H., et al., (2004), Optimization of radioimmunotherapy of renal cell carcinoma: labeling of monoclonal antibody cG250 with 131I, 90Y, $177 \mathrm{Lu}$, or $186 \mathrm{Re}$. Journal of Nuclear Medicine, Vol. 45, No. 2, pp. 327-337

[26] DeNardo, G. L., et al., (2004), Radiometals as Payloads for Radioimmunotherapy for Lymphoma Lymphoma. Clinical lymphoma, Vol. 5, pp. S5-S10

[27] Miller, W. H., et al., (2005), Evaluation of beta-absorbed fractions in a mouse model for 90Y, 188Re, 166Ho, 149Pm, 64Cu, and 177Lu radionuclides. Cancer biotherapy \& radiopharmaceuticals, Vol. 20, No. 4, pp. 436-449

[28] Bouchat, V., et al., (2010), Radioimmunotherapy with radioactive nanoparticles: biological doses and treatment efficiency for vascularized tumors with or without a central hypoxic area. Medical physics, Vol. 37, No. 4, pp. 1826-1839

[29] Häfeli, U., (2002), Radioactive microspheres for medical applications. In Physics and Chemistry Basis of Biotechnology, Springer Netherlands

[30] Campbell, A. M., Bailey, I. H. and Burton, M. A., (2000), Analysis of the distribution of intra-arterial microspheres in human liver following hepatic yttrium-90 microsphere therapy. Physics in medicine and biology, Vol. 45, No. 4, pp. 1023-1033

[31] Howell, R. W., Rao, D. V. and Sastry, K. S., (1989), Macroscopic dosimetry for radioimmunotherapy: nonuniform activity distributions in solid tumors. Medical physics, Vol. 16, No. 1, pp. 66-74

[32] Krane, S. K., (1988), Introductory Nuclear Physics, 2nd Ed., John Wiley \& Sons

[33] Carron, N. J., (2007), An Introduction to the Passage of Energetic Particles, Taylor \& Francis Group

[34] Ahlen, S. P., (1980), Theoretical and experimental aspects of the energy loss of relativistic heavily ionizing particles, Reviews of Modern Physics, Vol. 52, No. 1, pp. 121-173

[35] Demir, D., and Turşucu, A. (2012), Studies on mass attenuation coefficient, mass energy absorption coefficient and kerma of some vitamins. Annals of Nuclear Energy, Vol. 48, pp. $17-20$

[36] Ermis, E. E., and Celiktas, C., (2012), Determination of beta attenuation coefficients by means of timing method. Annals of Nuclear Energy, Vol. 41, pp. 115-118 
[37] Berger, M. J., (1973), Improved point kernels for electron and beta-ray dosimetry. Report NBSIR, pp. 73-107

[38] Bardies, M. and Chatal, J. F., (1994), Absorbed doses for internal radiotherapy from 22 beta-emitting radionuclides: beta dosimetry of small spheres. Physics in medicine and biology, Vol. 39, No. 6, pp. 961-981

[39] Pérez, P., et al., (2011), Dosimetry for beta-emitter radionuclides by means of Monte Carlo simulations. InTech. Chap. 11, pages 265-286

[40] Spencer L. V., (1959), Energy dissipation by fast electrons. Monograph No. 1; National Bureau of Standards

[41] Prestwich, W. V., Nunes, J. and Kwok, C. S., (1989), Beta Dose Point Kernels for Radionucides, Vol. 30, pp. 1036-1046

[42] Papadimitroulas, P., et al., (2012), A dose point kernel database using GATE Monte Carlo simulation toolkit for nuclear medicine applications: Comparison with other Monte Carlo codes. Medical physics, Vol. 39, No. 8, pp. 5238-5247

[43] Divoli, A., et al., (2009), Effect of patient morphology on dosimetric calculations for internal irradiation as assessed by comparisons of Monte Carlo versus conventional methodologies. Journal of Nuclear Medicine, Vol. 50, No. 2, pp. 316-323

[44] Berger, M. J., (1963), Monte Carlo calculation of the penetration and diffusion of fast charged particles. Methods in computational physics, Vol. 1, pp. 135-215

[45] Syme, A., McQuarrie, S. and Fallone, B. G., (2003), Beta dose-rate distributions in microscopic spherical tumors for intraperitoneal radioimmunotherapy. International Journal of Radiation, Oncology, Biology, Physics, Vol. 56, No. 5, 1495-1506

[46] Sgouros, G. and Hobbs, R. F., (2014), Dosimetry for Radiopharmaceutical Therapy. In Seminars in nuclear medicine, Vol. 44, No. 3, pp. 172-178

[47] Botta, F., et al., (2011), Calculation of electron and isotopes dose point kernels with FLUKA Monte Carlo code for dosimetry in nuclear medicine therapy. Medical physics, Vol. 38, No. 7, pp. 3944-3954

[48] Freudenberg, R., Wendisch, M. and Kotzerke, J., (2011), Geant4-Simulations for cellular dosimetry in nuclear medicine. Zeitschrift für Medizinische Physik, Vol. 21, No. 4, pp. 281-289

[49] Cross, W. G., Freedman, N. O. and Wong, P. Y., (1992), Beta-ray dose distributions from point sources in an infinite water medium. Health physics, Vol. 63, No. 2, pp. 160-171

[50] Zaidi, H. and Sgouros, G. (Eds.), (2010), Therapeutic applications of Monte Carlo calculations in nuclear medicine. CRC Press

[51] Sarfaraz, M. and Wessels, B. W., (1999), Validation of an analytical expression for the absorbed dose from a spherical $\beta$ source geometry and its application to micrometastatic radionuclide therapy. Clinical cancer research, Vol. 5, No. 10, pp. 3020s-3023s

[52] Leichner, P. K., (1994), A unified approach to photon and beta particle dosimetry. Journal of nuclear medicine: official publication, Society of Nuclear Medicine, Vol. 35, No. 10, pp. 1721-1729

[53] Fermi, E., (1950), Nuclear Physics, University of Chicago Press

[54] Fermi, E., (1934), An attempt of a theory of beta radiation, Z. Phys., Vol. 88, No. 161, pp. 10-21 
[55] Atkinson, W. J. and Brezovich, I. A., (2006), An analytic solution from a spherical gamma emitter, American Journal of Physics, Vol. 74, No. 12, pp. 1112-1114

[56] Kossert, K. and Schrader, H., (2004), Activity standardisation by liquid scintillation counting and half-life measurement of ${ }^{90} \mathrm{Y}$, Applied Radiation Isotopes, Vol. 60 pp. 741749

[57] Nilsson, S. B., (1956), On the Coulomb effect for the internal bremsstrahlung accompanying beta decay. Arkiv Fysik, Vol. 10

[58] Bé, M.-M., et al., (2006), Table of radionuclides (Vol. 3 - A=3 to 244). Monographie BIPM-5. Bureau International des Poids et Mesures, Sèvres, France

[59] Morita, M., (1963), Theory of Beta Decay. Progress of Theoretical Physics Supplement, Vol. 26, pp. 1-63

[60] Perkins, S. T., (1991), Tables and graphs of electron-interaction cross sections from 10 $\mathrm{eV}$ to $100 \mathrm{GeV}$ derived from the LLNL Evaluated Electron Data Library (EEDL). CA: Lawrence Livermore National Laboratory

[61] Rossi, B. B., (1952), High-energy particles, Vol. 952, New York: Prentice-Hall.

[62] Longmire, C., (1978), On the electromagnetic pulse produced by nuclear explosions. Antennas and Propagation, IEEE Transactions on, Vol. 26, No. 1, pp. 3-13

[63] Gurler, O. and Yalcin, S., (2005), A practical method for calculation of mass-attenuation coefficients of beta particles, Annals of Nuclear Energy, Vol. 32, pp. 1918-1925

[64] Thontadarya, S. R., (1985), Determination of range of beta particles in aluminum from their mass attenuation coefficients, The International Journal of Applied Radiation and Isotopes, Vol. 36, No. 3, pp. 251-252

[65] Sigel, H., (2004), Metal complexes in tumor diagnosis and as anticancer agents. CRC Press

[66] Osaka, G., at el., (1996), Pharmacokinetics, tissue distribution, and expression efficiency of plasmid [33P] DNA following intravenous administration of DNA/cationic lipid complexes in mice: use of a novel radionuclide approach. Journal of pharmaceutical sciences, Vol. 85, No. 6, pp. 612-618

[67] Siegel, J. A. and Stabin, M. G., (1994), Absorbed fractions for electrons and beta particles in spheres of various sizes. Journal of nuclear medicine: official publication, Society of Nuclear Medicine, Vol. 35, No. 1, pp. 152-156

[68] Cross, W. G., (1968), Variation of beta dose attenuation in different media. Physics in medicine and biology, Vol. 13, No. 4, pp. 611-618 